\title{
Cheminformatics-Aninsilico pharmacoinformatics approach in Drug Discovery
}

\author{
Nida Tabassum Khan* \\ Department of Biotechnology, Faculty of Life Sciences and Informatics, Balochistan University of Information Technology Engineering and \\ Management Sciences (BUITEMS) Quetta, Pakistan
}

Received: September 28, 2017; Accepted: October 24, 2017; Published: October 25, 2017

*Corresponding author: Department of Biotechnology, Faculty of Life Sciences and Informatics, Balochistan University of Information Technology Engineering and Management Sciences (BUITEMS) Quetta, Pakistan. E-mail: nidatabassumkhan@yahoo.com

\begin{abstract}
Cheminformatics is a novel in silico approach used in drug discovery and designing. Though it is in its budding stage but with the help of cheminformatics identification and analysis of structural and functional behavior of chemical compounds and biological molecules have been made easier. With such progress in the area of biochemistry, bioinformatics make use of such information in computer aided drug designing and development.
\end{abstract}

Key Words: Drug Discovery; Virtual Chemical Libraries; Open Babel; ChemReader

\section{Introduction}

Cheminformatics is a sub discipline of computer molecular science that deals with the information retrieval from chemical databases, three-dimensional molecular and crystal structure modeling, chemical reaction pathway prediction, molecular functional groups and docking sites [1].Cheminformatics originally emerged as a vehicle to help in drug discovery and development process. But now it has numerous application in numerous disciplines such as life sciences, chemistry, biochemistry, structural biology etc [2].This area of research mainly deals with small molecules, whereas bioinformatics covers genes, proteins, and other larger chemical compounds [3]. However both the disciplines are inter-related and quiet novel. Since Cheminformatics is a new field therefore still in its initial stages of development.

Following are some Cheminformatics tools mentioned below that aids in drug delivery and in its related developmental processes [4].

\section{Virtual Chemical Libraries}

Chemical databases and libraries contain suppository or imaginary compounds and structures (which do not exist in nature but there is a likelihood that these can be made).These guide the researchers suggesting different desired functionalities that do not exist yet [4]. Virtual libraries can contain information on possible synthesis methods and predicted stability of the different products that come into existence after going through a chemical pathway. It makes use of different chemical and physical principles to identify and choose the best chemical substance for a particular reaction or function, from large libraries of real and virtual molecules. The most suitable candidate can then be confirmed through wet lab experiments [5].

\section{Cheminformatics Tools in Drug Discovery}

The most prominent use of Cheminformatics is in drug discovery. The virtual libraries help predict the best substrate for a certain reaction leading to the wet lab experiments of drug discovery process. Before cheminformatics, it took decades for a drug to hit the market. But now with the help of Cheminformatics, this time period has been reduced immensely. Most of the unlikely candidates for a reaction are ruled out in the virtual screening process and then with the help of high throughput screening, a lot of time and manpower is saved [6].

\section{Symyx Draw}

It is a free chemical structure drawing program used to draw virtual diagrams of chemical substances. It uses its personal file format as well as the usual chemical file formats like MDL molfile, TGX file etc [7]. One can draw chemical structures and reactions, manually, an also using IUPAC names. It also provides the IUPAC names of the compounds that we have drawn. In addition to this, it allows search and retrieval through chemical databases [8].

\section{ChemDraws}

It is a structure drawing tool that allows one to create 3-D chemical structures and reactions with complete chains, rings, functional groups and bonds. It even allows one to find out the structures properties using the tool [9]. 


\section{ChemReader}

It is structure recognition software which retrieves chemical structure diagrams from digital pictures and converts them into computer readable chemical file formats. This information can then be used to search through chemical databases [10]

\section{JME Molecule Editor}

It is free java script software that allows users to draw and edit molecular diagrams along with creating substructure queries (queries regarding the molecular structures and its substructures). It can convert structures into numerous file formats [11].

\section{Wendi}

Wendi is a web engine for nonobvious drug information. A type of web based integrative data mining tool that is used to find associations between a query compound and scholarly publications, biological properties, genes and diseases that are usually ambiguous using multiple information sources [12].

\section{ChemMine Tool}

It is a tool that works online for analysis of small molecule and provides an interface between Cheminformatics and data mining tools for different analytical studies in chemical genomics and drug discovery [13].

\section{CML}

CML is chemical markup language, a combination of text and non textual information of chemical structure on the internet [14].

\section{PubChem}

It is a database for small molecules and their experimental biological activity. It combines and provides search, retrieval, visualization, analysis access tools [15].

\section{PLSR}

PLSR or Partial Least Squares Regression is a chemmetrics tool which relates and works on two matrixes on the basis of some predesigned models, and analyses data in the existence of many nonsensical and even incomplete variables [16].

\section{Open Babel}

It is a chemical format converting software that converts structures into different chemical formats [17].

\section{Conclusion}

Recognition and assessment of morphological and functional interactions of chemical compounds and biological molecules is one of the most important requirements for medical research which is made easier with the advent of Cheminformatics. A developing field which is progressing rapidly in the biochemical world.

\section{References}

1. Wishart D. S. Introduction to cheminformatics. Current protocols in bioinformatics. 2007:14.1.

2. Oprea TI, Tropsha A, Faulon JL, Rintoul MD. Systems chemical biology. Nature chemical biology. 2007; 3(8):447-450.

3. Olsson T, Oprea TI. Cheminformatics: a tool for decision-makers in drug discovery. Current opinion in drug discovery \& development. 2001;4(3):308-313.

4. Varnek A, Tropsha A. Cheminformatics approaches to virtual screening. Royal Society of Chemistry. 2008.

5. Xue L, Bajorath J. Molecular descriptors in chemoinformatics, computational combinatorial chemistry, and virtual screening. Combinatorial chemistry \& high throughput screening. 2000;3(5):363372 .

6. Oprea TI (Ed.). Chemoinformatics in drug discovery (Vol. 23). Weinheim: Wiley-VCH. 2005.

7. Martin E, Monge A, Duret JA, Gualandi F, Peitsch MC, Pospisil P. Building an R\&D chemical registration system. Journal of cheminformatics. 2012;4:11.

8. Kaushik M. A review of Innovative Chemical Drawing and Spectra Prediction Computer Software. Mediterranean Journal of Chemistry. 2014;3(1):759-766

9. Begam BF, Kumar JS. A study on cheminformatics and its applications on modern drug discovery. Procedia Engineering. 2012;38: 12641275 .

10. Park J, Rosania GR, Shedden KA, Nguyen M, Lyu N, Saitou K. Automated extraction of chemical structure information from digital raster images. Chemistry Central Journal. 2009;3(1):4.

11. Tetko IV, Gasteiger J, Todeschini R, Mauri A, Livingstone D, Ertl P, et al. Virtual computational chemistry laboratory-design and description. Journal of computer-aided molecular design. 2005;19(6):453-463.

12.Zhu Q, Lajiness MS, Ding Y, Wild D J. WENDI: a tool for finding nonobvious relationships between compounds and biological properties, genes, diseases and scholarly publications. Journal of cheminformatics. 2010;2(1):6.

13. Girke T, Cheng LC, Raikhel N. ChemMine. A compound mining database for chemical genomics. Plant physiology. 2005;138(2):573-577.

14. Phadungsukanan W, Kraft M, Townsend JA, Murray-Rust P. The semantics of Chemical Markup Language (CML) for computational chemistry: CompChem. Journal of cheminformatics. 2012;4(1):15.

15.Xie XQS. Exploiting PubChem for virtual screening. Expert opinion on drug discovery. 2010;5(12):1205-1220.

16. Fjell CD, Jenssen H, Hilpert K, Cheung WA, Pante N, Hancock RE, et al. Identification of novel antibacterial peptides by chemoinformatics and machine learning. Journal of medicinal chemistry. 2009;52(7):20062015.

17. O'Boyle NM, Banck M, James CA, Morley C, Vandermeersch T, Hutchison GR. Open Babel: An open chemical toolbox. Journal of cheminformatics. 2011; 3(1):33. 